\title{
THREE-DIMENSIONAL DIGITIZATION OF ENVIRONMENTS AND BUILDINGS FOR SMART CITY APPLICATIONS
}

\author{
C. Altuntas ${ }^{1} *$ \\ ${ }^{1}$ Konya Technical University, Faculty of Engineering and Natural Sciences, 42250 Selcuklu, Konya, Turkey - \\ caltuntas@ktun.edu.tr
}

KEY WORDS: Point cloud, Three-dimensional, Smart City, GIS, LiDAR, Photogrammetry.

\begin{abstract}
:
The smart cities that promise a sustainable future cannot be thought of independently from the spatial information infrastructure. It is very important to keep the spatial data infrastructure up-to-date for guidance and information in smart city applications (SCA). Easy and low-cost acquisition is an important factor in updating spatial data. Today, there are many measurement techniques to collecting 3-D spatial data of urban areas and land topography. On the other hand, indoor measurement and 3-D modelling techniques are used in the creation of building information modelling (BIM). In this study, measurement techniques that provide 3-D point cloud data to SCA are examined. Consequently, image based photogrammetry and dense matching methods enable low cost measurement than LiDAR based active measurement. The active 3-D measurement techniques have high accuracy especially for mid and long ranges. The LiDAR, that can be applied at day or night time, offer more opportunity to performing SCA like autonomous vehicle and robotic navigations. Nevertheless, LiDAR can only capture structure, not texture, and therefore has limits to the types of data that it can capture. The LiDAR and image based methods are complement to each other in 3-D reality capture. The 3-D measurement techniques are exploited according to SCA as alone or together.
\end{abstract}

\section{INTRODUCTION}

The rapid increase in the population and the many humanitarian needs make it difficult to meet the demands. Smart cities are a set of services that offer effective solutions to meeting human needs and managing resources. The geographic information system (GIS) is one of the basic structures of smart city ( $\mathrm{Li}$ et al., 2019). GIS are also called as urban information systems for urban areas. The building information modelling (BIM) has been used to manage information about building and structure processes. SCA that include GIS and BIM integration offer more alternatives in meeting demands and performing tasks (Garramone et al., 2020).

The spatial data that is a component of GIS are obtained from various sources. Orthophoto maps, which are obtained from high resolution aerial photographs, are the basis of geographic information systems. A 3-D city model is created on this basic structure. Digital elevation model (DEM) obtained from LiDAR data is also used in the three-dimensional (3-D) modelling of urban details. The buildings 3-D representation in information systems is performed in three categories from level of detail (LoD1) to LoD3 according to the level of detail (Biljecki et al., 2016). Every up level gets a little closer to the real look and consist of more details. LoD4 level is a 3-D visualization that includes interior details such as wall, floor, roof, window, door etc. (Fan and Meng, 2009). A BIM requires measuring the interior details and creating a 3-D model (Garramone et al., 2020).

Time-of-flight (ToF) camera, terrestrial laser scanner (TLS) and backpack mobile LiDAR techniques are usually used to measure building indoor details (Wang et al., 2018). Mobile mapping systems are exploited to obtain street view urban details (Nebiker et al., 2021). On the other hand, oblique aerial photographs are used for modelling the building facades (Toschi et al., 2017). Terrestrial laser scanning and photogrammetric method complete the occlusion areas that cannot be measured with these techniques (Anton et al., 2019). As can be seen, different measurement techniques are used in the creation of spatial data infrastructure of geographic information systems. The obtained 3-D point cloud data is combined in a common coordinate system, giving a structure for query and analyse. In this study, 3-D point cloud acquisition methods of spatial information systems, which form the basis of smart cities, were examined and analysed.

\section{SMART CITY}

A smart city is a set of systems in which current technology is used at the highest level in order to meet the requirements effectively and efficiently with the existing resources and infrastructure facilities. The rapid increase in the population necessitates the efficient use of existing resources. At this stage, smart cities aim the feature as an environmentally, socially and economically sustainable. On the other hand, it provides more alternative and opportunities to the decision makers responsible for realizing these goals.

The smart cities are generated on spatial data, communication and software structures. GIS constitutes the data infrastructure (Yang, 2019; Shirowzhan et al., 2020). The geospatial data is collected by many techniques. The spatial data must respect every change in the city in order to smart city services to be sustainable. The smart cities also provide various data from different sources such as sensors that measure air pollution, noise and water quality. The communication in smart cities refers to internet possibilities (IoT) in executing certain tasks (Kim et al., 2017; Syed et al., 2021). This communication can be between man-machine or machine-machine. The human interaction with the smart city is provided by mobile phone or mobile devices. Thanks to the communication platform, services such as driverless vehicles, remote control of home services and smart transportation systems are performed. The software unit is 
an important component that ensures the most effective use of existing resources while meeting the demands (Dominguez et al., 2020). The quality of many services will be increased with software that have artificial intelligence applications by the time.

The smart cities should be designed to provide the most information about any city place or location to users. The aim is manage the people and making things safer or more environmentally. Thus, the data is collecting through sensors and systems in order to measure and monitor conditions throughout the city. The collected data are then analysed and turned into information that is assist people to make more informed decisions, and intelligence to machines to take actions.

\section{3-D POINT CLOUD MEASUREMENT TECHNIQUES}

\subsection{Aerial LiDAR}

The topography of the earth are digitized with LiDAR sensors placed on aerial vehicles such as airplane or helicopter. LiDAR sensors are widely used in 3D survey of urban and rural areas. The aerial LiDAR makes measurements from a height of between $300-5000 \mathrm{~m}$ with the accuracy of $15 \mathrm{~cm}$ horizontally and $5 \mathrm{~cm}$ vertically. The on the fly GPS data enables georeferenced measurement directly from LiDAR. Low height flights (300-400 m altitude) are performed with fixed-wing or helicopter (Höfle and Hollaus, 2010). High density (70 points $/ \mathrm{m}^{2}$ ) air LiDAR data is used for urban planning, building modelling and disaster management purposes. LiDAR data density for urban 3-D modelling can reach up to 500 points $/ \mathrm{m}^{2}$. Low-density (1-15 points $/ \mathrm{m}^{2}$ ) LiDAR data from higher (up to $3000 \mathrm{~m}$ ) level flights is used to measure larger areas at the national level (Stanley and Leafer, 2021). The multiple return signals from LiDAR in vegetated areas provide additional information such as tree heights and volumes in addition to DEM.

\subsection{Aerial Photogrammtry}

The photogrammetric aerial cameras provide very high resolution images. The ground resolution of photogrammetric images varies depending on flight altitude and camera pixel dimensions and is between $5-40 \mathrm{~cm}$. Aerial photogrammetry is especially exploited for 3-D modelling, orthophoto production and DEM generation of urban areas (Figure 1). On the other hand, an oblique camera is better especially in modelling facades of buildings in urban areas (Haala et al., 2015). In aerial photogrammetry, the spatial measurement accuracy depends on the image scale, and is better than $10 \mathrm{~cm}$. The georeferencing of measurements can be done based on flight GPS data, or ground control points. The aerial photogrammetry is very suitable for mapping of large areas.

The photogrammetric processes have become much easier with adapting the developments in computer technology. Thus, 3-D point cloud data can be generated from photogrammetric images automatically. The method, which creates a point cloud from the photogrammetric images, is called SfM photogrammetry. Thanks to the SfM technique, georeferenced orthophotos and DEM can be obtained from aerial photogrammetry in a very short time (Cao et al., 2019). In smart cities, LoD2 city models can be generated from aerial photogrammetric point cloud. SfM photogrammetry is convenience to urban planning, and disaster monitoring in a short time, and also mobile mapping.

\subsection{Unmanned Aerial Vehicle (UAV)}

UAVs are low cost instruments that can be used with on board sensors and cameras. It has been used for land surveying, air pollution control, traffic monitoring, disaster monitoring and etc. in smart city (Mohammed et al., 2014; Giyenko and Cho, 2016). The legal regulations for the use of drones have increased its implementation for various aims. The main usage areas of UAVs in SCA are discussed below.

Topographic surveying and 3-D modelling: UAVs have been exploited for many terrestrial measurements. 3-D dense point cloud model is generated from UAV images by using SfM algorithm. The SfM is performed easily without any technical knowledge about the photogrammetry. UAV images are high resolution and the ground sampling distance can be better than $5 \mathrm{~cm}$. The sub-millimetre accuracy is achieved from the photogrammetric evaluation of UAV based photographs.

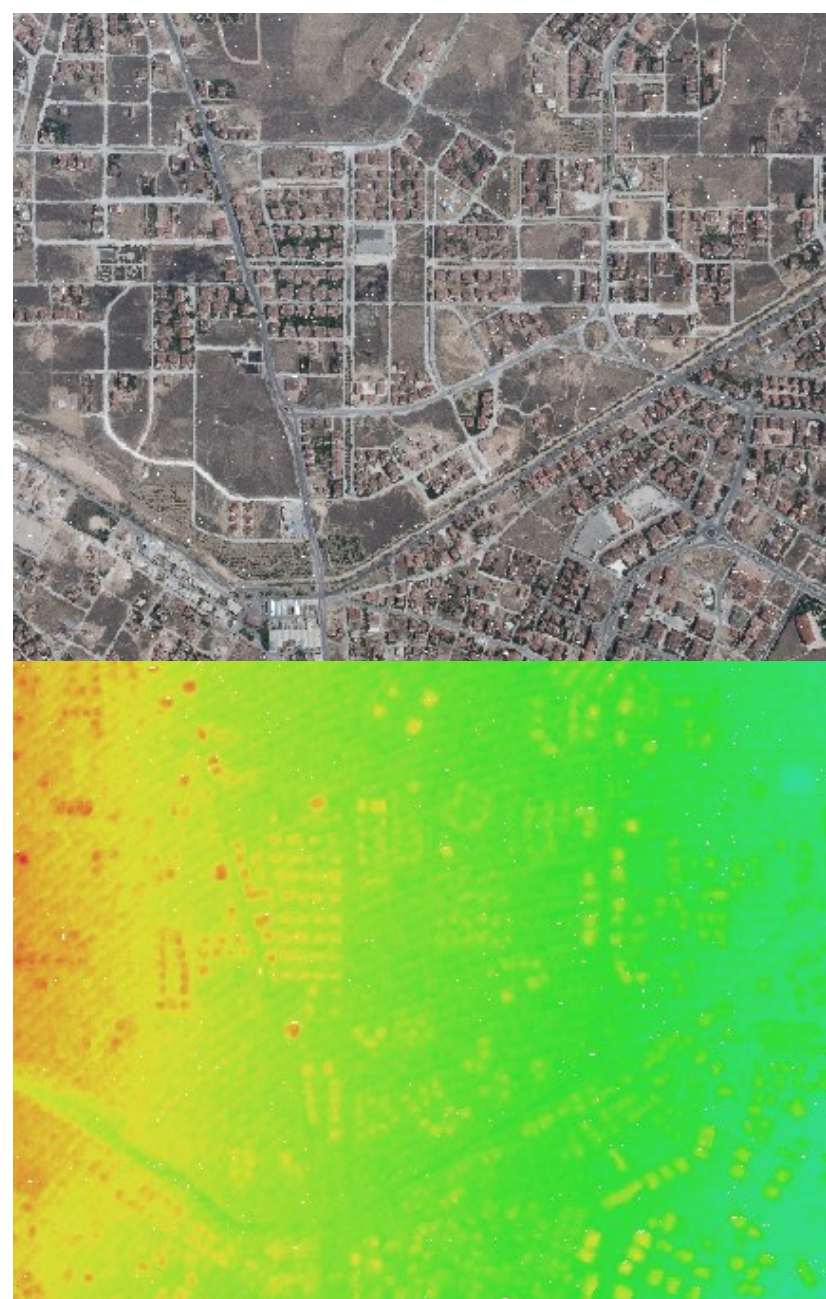

Figure 1. Orthophoto and DEM from aerial photogrammetric images.

Inspection: UAV is very useful when it is necessary to monitor the changes by making measurements at certain periods. Unauthorized constructions and other physical changes in urban areas can be detected by comparing the point clouds to be created from the UAV images. In this respect, UAVs are an important instrument to get spatial or non-spatial data especially for urban decision makers. 
Agriculture and environmental management: UAVs can monitor agricultural product development with proper equipment such as thermal cameras. On the other hand, tree heights and types can be determined from the UAV LiDAR data and camera images. Furthermore water quality and air pollution can be measured by an UAV equipped with an appropriate sensors. The tree species in forest can be classified by using UAV images and other proper sensor data.

Monitoring vehicle and pedestrian traffic: In smart cities, the duration of traffic lights should be adjusted according to vehicle density. Traffic lights can be controlled by monitoring heavy traffic routes with an UAV at certain times or continually. On the other hand, pedestrian traffics can also be monitoring. By sharing traffic data with mobile smart phones in smart cities, alternative routes can be offered for vehicles and pedestrians. It can be also ensured that local distributors reach their destination in a shorter time.

Disaster management: Damages of natural disasters such as fire, flood, earthquake can be obtained very quickly from the UAV sensors to manage the first aid to the certain locations. The forest fires or direction of the fire can be predicted from UAV images. In case of floods, dangerous areas can be estimated from UAV based DTM models as well taking into account geodetic data. In addition, landslides and other natural disasters in inaccessible areas can be viewed.

Security applications: Thanks to the developments in computer technologies, target tracking can be done from photographs. A criminal person can be identified from public domain images.

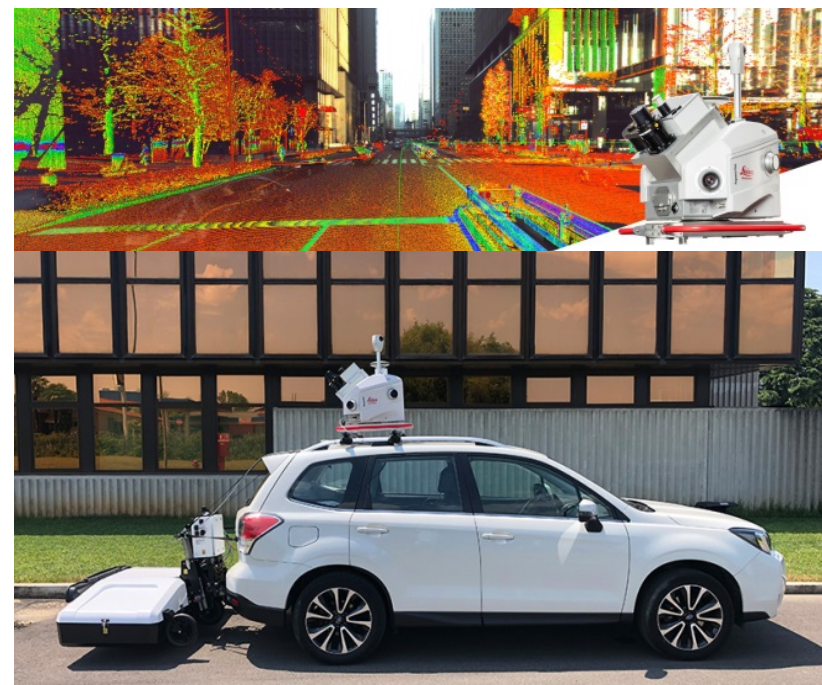

Figure 2. Leica Pegasus mobile mapping system (Leica Geosystems, 2021).

\subsection{Mobile Mapping}

Mobile mapping refers to the collection of spatial data by using mobile vehicle, backpack or watercraft. The vehicles are equipped with the LiDAR, camera and other related instruments to getting street view 3-D data. It enables high-resolution point cloud measurement of urban details that cannot be measured from the aerial methods (Figure 2). SCA are possible with GIS databases where urban details are completely represented in digital environment. 3-D mobile measurement data are transferred into information systems. The camera images acquired with LiDAR data provide colour and texture data. Mobile LiDAR data provides a 3-D panoramic view at the current point. The complete 3-D modelling of urban details is important for infrastructure applications, route selection, visualization of structures and traffic. In smart cities, various inquiries can be made thanks to the 3-D model in georeference system.

The areas where the vehicles cannot reach in the urban area are measured with a laser that can be carried in a backpack (Figure 3 ). Integration of the measurements where GPS signals cannot be received is provided by simultaneous localization and mapping (SLAM) method. The mobile surveying is also performed with marine vehicles to display the cost line topography and buildings.
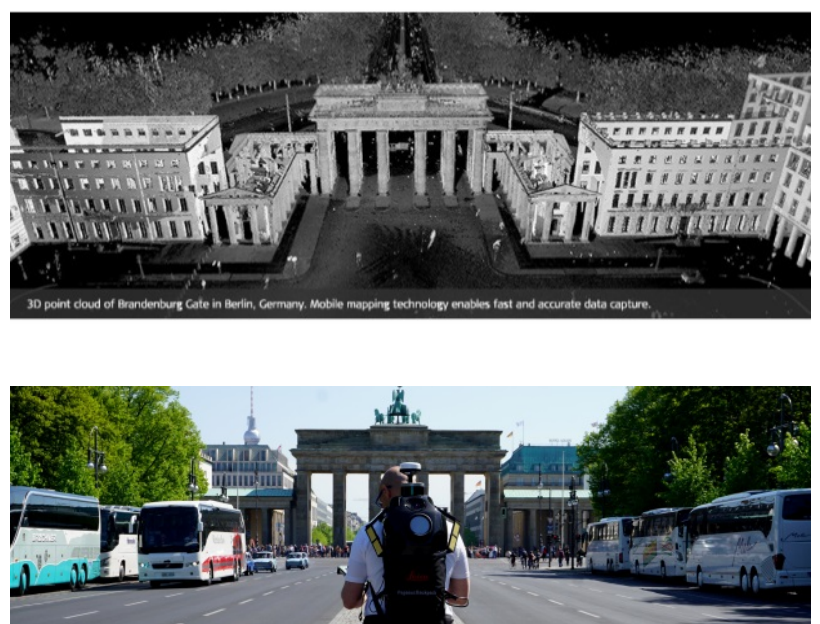

Figure 3. Backpack mobile measurement (Leica Geosystems, 2021).

\subsection{TLS}

TLS is a static measurement technique to getting rich spatial data for SCA. It is very useful digitization technique for indoor and street level details. Indoor measurement includes object and structure digitization for BIM applications, which are an important component of smart city. TLS collects both semantic and geometric data as a dense point cloud in millimetre level accuracy (Figure 4). In addition, it is used for detecting deformations and changes that require measurement at certain periods (Sepasgozar et al., 2016). In addition, the completion status of the constructions can be determined as a result of the comparison of certain period TLS point clouds (Sepasgozar et al., 2015)

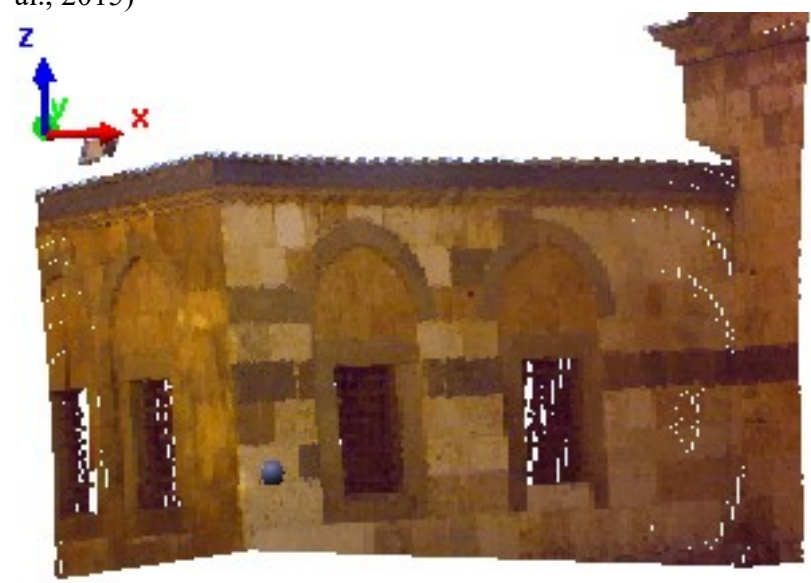

Figure 4. TLS point cloud data of building façade. 


\subsection{ToF Camera}

Tof camera makes measurements at video rates. It is widely used in robotic applications and movement detection around the vehicle. In addition, it is used to ensure the safety of workers in a factory site at continuous measurement mode. In SCA, measurement data analysis and information extraction are also very important. In particular, the observation of crowded areas and the analysis of human movements are possible from the camera data. Interior details in BIM applications are measured with static mode or mobile robots to creation LoD4 building models. On the other hand, ToF camera can be used to monitoring and managing the production lines at the factories in smart city.

\subsection{Flash LiDAR}

Flash LiDAR camera is widely used to route detection for driverless vehicles. The smart cities in the future, vehicles will be able to be managed without drivers. Thanks to the flash LiDAR camera placed in the vehicles, details up to $500 \mathrm{~m}$ away can be measured instantly with sufficient point density and accuracy. Flash LiDAR measurement speed is compatible with the speed of vehicles and is very effective in urban applications. The braking distance and speed of the vehicles will be determined with the flash LiDAR data. In this way, traffic accidents will be prevented in smart cities. This will make roads safer for vehicles and pedestrians. Flash LiDAR can be used for street view 3-D modelling, traffic monitoring and driverless vehicle management in urban area.

The vehicles will be in communication with other vehicles and the infrastructure in the smart cities of the future. Flash LiDAR measurement has an important place in interactions between vehicles. Flash LiDAR can be used in all weather conditions, day or night. It is also used for tracking and guiding unmanned aircraft. Thus, unmanned flights will be possible and accidents of aircraft will be prevented. Taxi service will be provided by unmanned ground vehicles (UGVs) or UAVs.

The hybrid laser scanners, which have common technical specifications with terrestrial laser scanner (mechanical scanner) and flash LiDAR, also enable to panoramic mobile measurement (Figure 5). This makes safer to the road for autonomous vehicles and pedestrians.

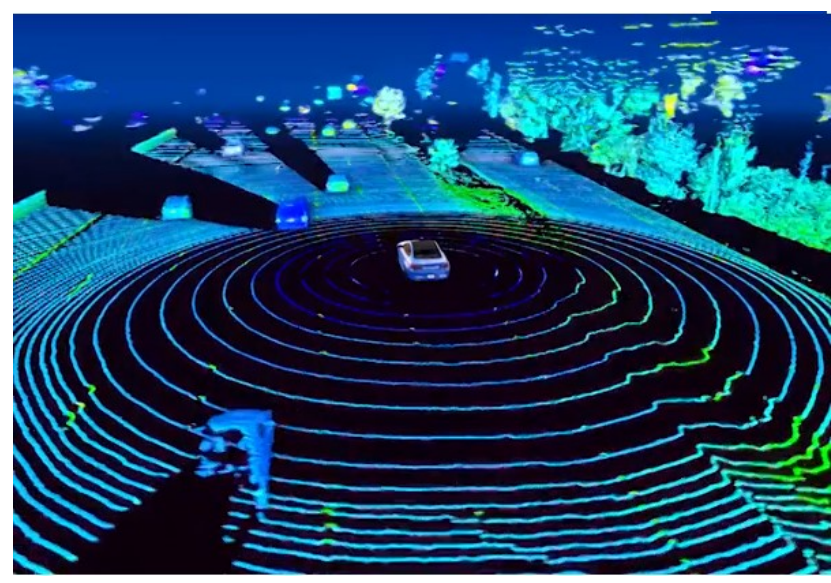

Figure 5. Panoramic LiDAR measurement in motion on the road (Velodyne Lidar, 2021)

\subsection{Terrestrial Photogrammetry}

Close-range photogrammetric images provide rich texture information in addition to spatial data. This information is useful to classify and identify objects (Niederheiser et al., 2021). Terrestrial photographs are taken with amateur cameras or mobile phone cameras, and point cloud is created by SFM algorithm. Therefore, it is a low-cost and easy measurement technique. The unmeasured parts of urban area and building details can be completed with this method (Figure 6). Moreover, the location information of the camera can be used to georeferencing of the created dense point cloud. The building facades in the 3-D modelling of cities can be modelled by terrestrial photogrammetry with high resolution.

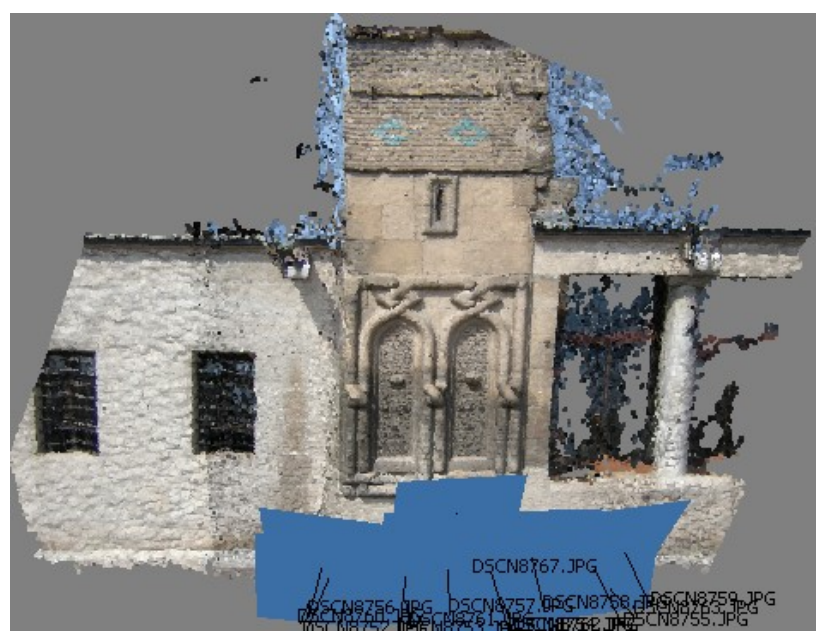

Figure 6. SfM based point cloud from terrestrial images.

\section{DISCUSSION}

LiDAR is a key technology for smart cities. It creates accurately, autonomously, efficient smart city solutions about people and vehicles across large areas. LiDAR based 3-D point cloud data provides both spatial location and depth information that can be used to identify, classify, and track moving objects.

The 3-D measurement techniques collect image and spatial data. The protecting citizen personal data for security reason can be problem via the measurement data. LiDAR sensors collect no personally identifying information, protecting the privacy and anonymity of citizens. It collects spatial data and reflectance from the environment only. This enables protecting privacy and optimizing necessary security resources for cities and commercial building planners.

The LiDAR enables to collecting reliable data for outdoor and indoor measurement. The measurement is possible in day or night at any weather conditions. Furthermore flash LiDAR and ToF camera makes measurement to motion objects. This ensures continuously data capture at all times and across all locations throughout the city.

The SfM photogrammetry enables to generation 3-D point cloud data for non-specialist persons by using any camera images, especially smart phone. On the other hand many smart phones have additional sensors such as infrared or thermal camera. Thus, more information can be collected from the location, and it enables more efficient decision for smart city interactions. 
Non of the point cloud measurement method makes complete measurement for 3-D modelling in SCA. Usually, two or three methods are used together in 3-D modelling such as oblique photogrammetry and aerial LiDAR in CityMapper2. Full 3-D city modelling requires combination of aerial and terrestrial measurements. The main application areas of 3-D measurement techniques for smart cities are given on Table 1 and Figure 7. The integration of many source point cloud data can be made by georeferencing for outdoor. The point cloud-to-point cloud registration can also be executed for the integration especially for indoor data.

Smart city makes digitization of information and public services. 3-D point cloud measurement techniques enable to getting high density spatial data for 3-D visualization and spatial analysis. More analysis is possible from point cloud data with segmentation and classifications. At this step, the software components of the smart city has important role.

Table 1. The main properties of 3-D measurement techniques for SCA.

\begin{tabular}{|l|l|l|l|l|l|}
\hline & $\begin{array}{l}\text { Operation } \\
\text { time }\end{array}$ & $\begin{array}{l}\text { Usage } \\
\text { environments }\end{array}$ & $\begin{array}{l}\text { Max. } \\
\text { Range[m] }\end{array}$ & $\begin{array}{l}\text { Continuously } \\
\text { measurement? }\end{array}$ & Possible application areas \\
\hline Aerial LiDAR & Daytime/Night & Outdoor & 5500 & No & $\begin{array}{l}\text { 3-D building model, SYM, 3- } \\
\text { D city modelling }\end{array}$ \\
\hline $\begin{array}{l}\text { Aerial } \\
\text { photogrammetry }\end{array}$ & Daytime & Outdoor & 10000 & No & $\begin{array}{l}\text { 3-D building model, SYM, } \\
\text { terrain mapping }\end{array}$ \\
\hline UAV & Daytime & Indoor/Outdoor & 300 & No & $\begin{array}{l}\text { Change detection, Disaster } \\
\text { monitoring, Topography } \\
\text { measurement }\end{array}$ \\
\hline Mobile mapping & Daytime & Outdoor & 250 & No & $\begin{array}{l}\text { Street view 3D modelling, } \\
\text { Shore line monitoring }\end{array}$ \\
\hline TLS & Daytime & Indoor/Outdoor & 6000 & No & Building and Object modelling \\
\hline ToF camera & Daytime & Indoor & 10 & Yes & $\begin{array}{l}\text { Robotic and LoD4 BIM } \\
\text { measurement, Surveilance }\end{array}$ \\
\hline Flash LiDAR & $\begin{array}{l}\text { Daytime/ } \\
\text { Night }\end{array}$ & Indoor/Outdoor & 1000 & Yes & $\begin{array}{l}\text { Autonomous } \\
\text { Surveilance, Crowd and traffic } \\
\text { monitoring, 3D mapping }\end{array}$ \\
\hline $\begin{array}{l}\text { Terrestrial } \\
\text { photogrammetry }\end{array}$ & Daytime & Indoor/Outdoor & 500 & No & Building and Object modelling \\
\hline
\end{tabular}

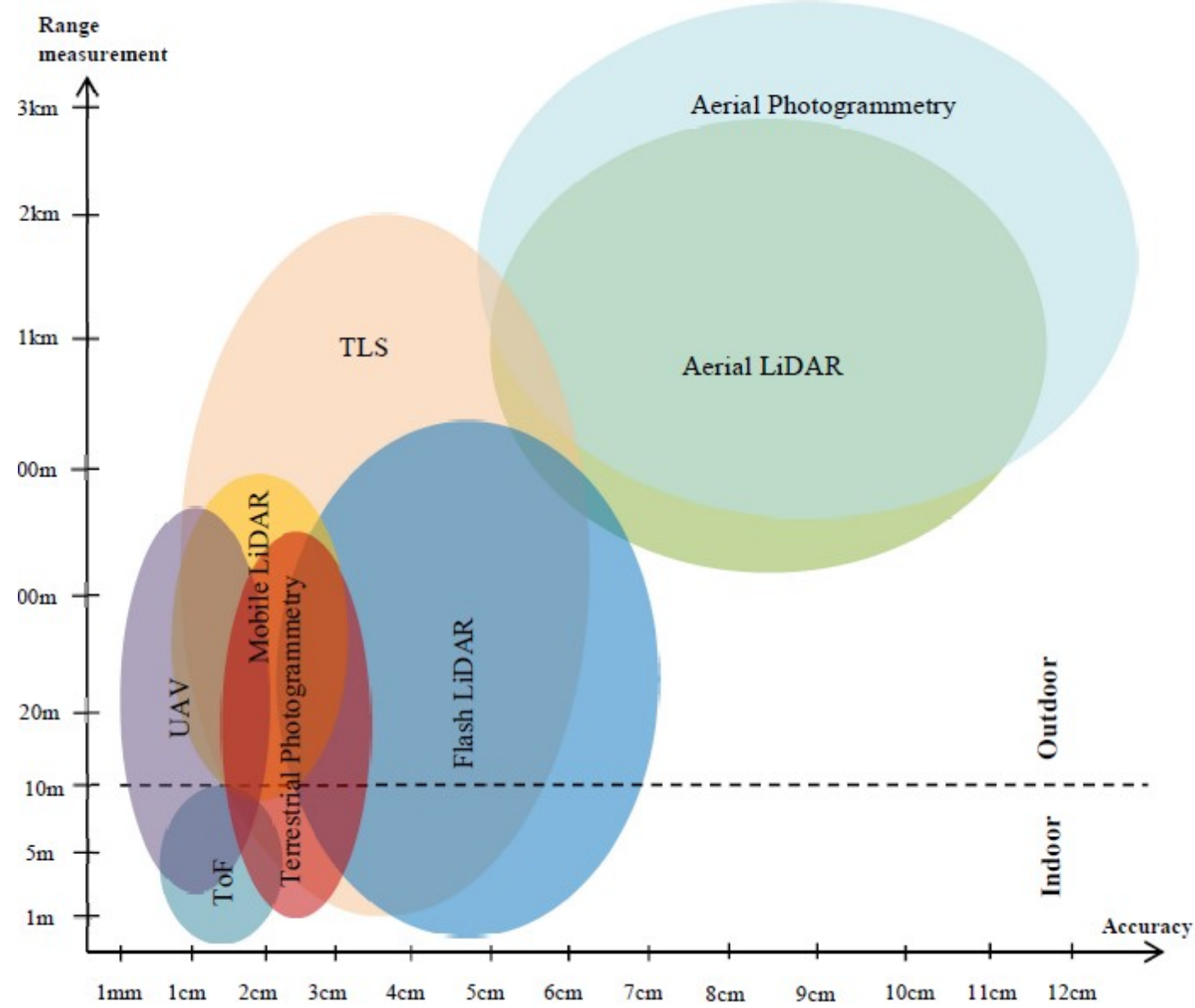

Figure 7. The specific characteristics of point cloud measurement techniques proposed for SCA. 


\section{CONCLUSION}

Smart city spatial applications hugely depended to GIS database. Today there are many techniques to make point cloud measurement with high density. The physical environments are a topic to change by construction, natural hazards, and other effects. Thus the GIS database must be updated by measuring these changes. The aerial based measurement techniques are proper for large areas in which urban or suburban with high cost. However terrestrial techniques are suitable for street based modelling and object measurement. The aerial and terrestrial measurement methods are complement to each other for digitization of the city environments. The more spatial data enables more information and facility to public and decision makers by making smarter of city.

The LiDAR based measurement techniques offer more opportunity to SCA like autonomous vehicle and robotic navigations. Active 3-D measurement techniques enable high accuracy especially for mid and long range measurements. On the other hand, demands of fast, high density and accuracy on the measurement increases the capacity of data processor units and budget of the instruments.

\section{REFERENCES}

Anton, D., Pineda, P., Medjdoub, B., Iranzo, A. 2019. As-built 3D heritage city modelling to support numerical structural analysis: Application to the assessment of an archaeological remain. Remote Sensing, 11(11), 1276. doi.org/10.3390/rs11111276.

Biljecki, F., Ledoux, H., Stoter, J., Vosselman, G., 2016. The variants of an LOD of a 3D building model and their influence on spatial analyses. ISPRS Journal of Photogrammetry and Remote Sensing, 116, 42-54.

Cao, L., Liu, H., Fu, X., Zhang, Z., Shen, X., Ruan, H., 2019. Comparison of UAV LiDAR and digital aerial photogrammetry point clouds for estimating forest structural attributes in subtropical planted forests. Forests, 10(2), 145. doi.org/10.3390/f10020145

Dominguez, J.M.L., Al-Tam, F., Sanguino, T.J.M., Correia, N., 2020. Analysis of machine learning techniques applied to sensory detection of vehicles in intelligent crosswalks. Sensors, 20(21), 6019. doi.org/10.3390/s20216019.

Fan, H., Meng, L., 2009. Automatic derivation of different levels of detail for 3D buildings modeled by CityGML. 24th International Cartography Conference, Santiago, Chile, pp. 1521.

Garramone, M., Moretti, N., Scaioni, M., Ellul, C., ReCecconi, F., Dejaco, M. C., 2020. BIM and GIS integration for infrastructıre assest management: A bibliometric analysis. ISPRS Ann. Photogramm Remote Sens. Spatial Inf. Sci., VI4/W1-2020, 77-84. doi.org/10.5194/isprs-annals-VI-4-W12020-77-2020.

Giyenko, A., Cho, Y.I., 2016. Intelligent UAV in smart cities using IoT. 16th International Conference on Control, Automation and Systems, Oct. 16-19, Gyeongju, Korea, pp. 207-210. doi.org/10.1109/ICCAS.2016.7832322.
Haala, N., Rothermel, M., Cavegn, S., 2015. Extracting 3D urban models from oblique aerial images, The Biennial International Joint Urban Remote Sensing Event, pp. 1-4, doi.org/10.1109/JURSE.2015.7120479.

Höfle, B., Hollaus, M., 2010. Urban vegetation detection using high density full waveform airborne lidar data-combination of object-based image and point cloud analysis. Int. Arch. Photogramm Remote Sens. Spatial Inf. Sci., XXXVIII-7B, 281286.

Kim, T. H., Ramos, C., Sabah, M., 2017. Smart city and IoT. Future Generation Computer Systems, 76, 159-162.

Leica Geosystems, 2021. Mobile Sensor Platforms. leicageosystems.com/products/mobile-sensor-platforms/storiesinsights/digitising-environments (Access: 9 August 2021).

Li, W., Batty, M., Goodchild, M. F., 2019. Real-time GIS for smart cities. International Journal of Geographical Information Science, 34(2), 311-324.

Mohammed, F., Idries, A., Mohamed, N., Al-Jaroodi, J., Jawhar, I., 2014. UAVs for smart cities: Opportunities and challenges, International Conference on Unmanned Aircraft Systems, May 27-30, Orlando, FL, USA, pp. 267-273. doi.org/10.1109/ICUAS.2014.6842265.

Nebiker, S., Meyer, J., Blaser, S., Ammann, M., Rhyner, S. 2021. Outdoor mobile mapping and AI-based 3D object detection with low-cost RGB-D cameras: The use case of onstreet parking statistics. Remote Sensing, 13(16), 3099. doi.org/10.3390/rs13163099.

Niederheiser, R., Winkler, M., Di Cecco, V., et al., 2021. Using automated vegetation cover estimation from close-range photogrammetric point clouds to compare vegetation location properties in mountain terrain. GIScience and Remote Sensing, 58(1), 120-137.

Sepasgozar, S., Lim, S., Shirowzhan, S., Kim, P., Nadoushani, Z.M. 2015. Utilisation of a new terrestrial scanner for reconstruction of as-built models: A comparative study. International Symposium on Automation and Robotics in Construction, 15-18 June, Oulu, Finland. doi.org/10.22260/ISARC2015/0027.

Sepasgozar, S. M. E., Forsythe, P. J., Shirowzhan, S. 2016. Scanners and photography: A combined framework. 40th Australasian Universities Building Education Association Conference, 6-8 July, Cairns, Australia, pp. 819-828.

Shirowzhan, S., Tan, W., Sepasgozar, S.M.E. 2020. Digital twin and cyberGIS for improving connectivity and measuring the impact of infrastructure construction planning in smart cities. ISPRS Int. J. Geo-Inf., 9(4), 240. doi.org/10.3390/ijgi9040240.

Stanley, M. H., Laefer, D. F., 2021. Metrics for aerial, urban LiDAR point clouds. ISPRS Journal of Photogrammetry and Remote Sensing, 175, 268-281. doi:10.1016/j.isprsjprs.2021.01.010.

Syed, A. S., Sierra-Sosa, D., Kumar, A., Elmaghraby, A., 2021. IoT in smart cities: A survey of technologies, practices and 
challenges. Smart Cities, 4(2), 429-475. doi.org/10.3390/smartcities4020024.

Toschi, I., Ramos, M. M., Nocerino, E., Menna, F., Remondino, F., Moe, K., Poli, D., Lrgat, K., Fassi, F., 2017. Oblique photogrammetry supporting 3D urban reconstruction of complex scenarios. Int. Arch. Photogramm Remote Sens. Spatial Inf. Sci., XLII-1/W1, pp. 519-526. doi.org/10.5194/isprs-archives-XLII-1-W1-519-2017.

Velodyne Lidar, 2021. Alpha Prime. velodynelidar.com/products/alpha-prime/ (Access: 9 August 2021).
Wang, C., Hou, S., Wen C., Gong, Z., Li, Q., Sun, X., Li, J., 2018. Semantic line framework-based indoor building modeling using backpacked laser scanning point cloud. ISPRS Journal of Photogrammetry and Remote Sensing, 143, 150-166.

Yang, B. 2019. Developing a mobile mapping system for 3D GIS and smart city planning. Sustainability, 11(13), 3713. doi.org/10.3390/su11133713. 